\title{
Cancer mortality among workers in the German rubber industry: 1981-91
}

Stephan K Weiland, Kenneth A Mundt, Ulrich Keil, Barbara Kraemer, Thomas Birk, Michael Person, Annette M Bucher, Kurt Straif, Jutta Schumann, Lloyd Chambless

\begin{abstract}
Objectives-To determine the cancer specific mortality of active and retired workers of the German rubber industry with emphasis on cancer sites which have been associated with the rubber industry in previous studies.

Methods-A cohort of 11663 German men was followed up for mortality from 1 January 1981 to 31 December 1991. Cohort members were active $(n=7536)$ or retired $(n=4127)$ at the beginning of the study, and had been employed for at least one year in one of five study plants producing tyres or general rubber goods. Vital status was ascertained for $99.7 \%$ of the cohort members, and cause of death found for $96.8 \%$ of the 2719 decedents. Age and calendar year adjusted standardised mortality ratios (SMR) and 95\% confidence intervals (95\% CI) were calculated overall from national reference rates and stratified by year of hire and by years since hire.
\end{abstract}

Results-Mortalities from all causes (SMR 108; 95\% CI 104-112) and all cancers (SMR 111; 95\% CI 103-119) were significantly increased in the study cohort. Significant excesses in the mortalities from lung cancer (SMR 130; 95\% CI 115-147) and pleural cancer (SMR 401; 95\% CI 234-642) were identified. SMRs higher than 100 were found for cancers of the pharynx (SMR 144; 95\% CI 76-246), oesophagus (SMR 120; 95\% CI 74-183), stomach (SMR 110; 95\% CI 86-139), rectum (SMR 123; 95\% CI 86-170), larynx (SMR 129; 95\% CI 69-221), prostate (SMR 108; 95\% CI 84-136), and bladder (SMR 124; 95\% CI 86-172), as well as for leukaemia (SMR 148; 95\% CI 99-213). Mortalities from liver cancer, brain cancer, and lymphoma were lower than expected.

Conclusions-Mortalities from cancer of several sites previously associated with the rubber industry were also increased among workers of the German rubber industry. Results of the stratified analyses are consistent with a role of occupational exposure in the aetiology of some of these cancers.

(Occup Environ Med 1996;53:289-298)

Keywords: cancer mortality; rubber industry; cohort study; occupational epidemiology
Health effects of exposures sustained by workers in the rubber industry have been studied in several relatively large epidemiological investigations. Studies carried out in Europe, ${ }^{1-14}$ the United States, ${ }^{15-26}$ and Asia ${ }^{27-28}$ have reported increased mortality or morbidity from cancers of the bladder, lung, stomach, and several other sites, as well as from leukaemia. However, no consistent excess from any of these malignant neoplasms has been found across the various studies or countries. Rubber workers are potentially exposed to several carcinogens-for example, aromatic amines, nitrosamines, polycyclic aromatic hydrocarbons (PAH), solvents, and asbestos. ${ }^{29-34}$ The exposure conditions in the rubber industry, however, are very complex and highly variable between work sites and plants, and over time. ${ }^{29}{ }^{35}$ It is therefore difficult to attribute the observed health impairments to specific exposures, and only few specific associations-for example, bladder cancer and aromatic amines, leukaemia and solvents ${ }^{29}{ }^{30}$ - have been widely accepted.

In the late $1980 \mathrm{~s}$, German occupational physicians raised concerns about a possible increase in morbidity and mortality from laryngeal and other cancers among workers in the German rubber industry. At that time, no epidemiological data on workers in the German rubber industry were available. As the need to document the current and future health of rubber workers was raised also by the industry, it was decided in 1990 to initiate a major epidemiological investigation, which would ultimately serve several epidemiological purposes. ${ }^{36} 37$ In the present paper we report the study methods, present the total and cause specific mortality experience of production workers in the German rubber industry, and examine the cancer specific mortality by time related factors.

\section{Methods}

GERMAN RUBBER INDUSTRY AND STUDY POPULATION

In 1992 more than $50 \%$ of about 95000 employees of the German rubber industry worked in factories with at least 1000 employees. Of these, $70 \%$ were blue collar workers. For practical reasons, the present study included large factories in a few geographic locations, in which the data for the identification of potential cohort members and the reconstruction of their employment histories were of adequately high quality. Available data 
sources were evaluated during a pilot phase of nine months, and five factories belonging to three different companies were selected. Each of the five study plants employed more than 1000 production workers, had produced tyres or general rubber goods since the first half of this century, and was located in one of three different states (Bundesländer) of Germany.

To have reasonable statistical power to consider relatively rare causes of death, including several specific cancers, a large cohort was required. With the limited resources available, enumeration of a cohort with the necessary size required access to electronic data. Because personnel data files at study plants contained only information on active employees or those who had left within the previous two years, additional sources of information were sought. The only feasible alternative was computer files maintained by the health insurance companies affiliated to the rubber companies (Betriebskrankenkassen). These files contained information on all men who had been insured since 1980 , including those who died or left the company. Because legislation until 31 December 1991 required that all blue collar workers obtain health insurance from these companies (in contrast to white collar workers, who could obtain coverage elsewhere), the study population was restricted to blue collar workers, who were also more likely to have sustained occupational exposures relevant to health. Through comparisons with the active workforce of the rubber companies, it was estimated that about $99 \%$ of the blue collar workers in the factories actually had health insurance through these companies, assuring that the enumeration of cohort members was reasonably comprehensive.

\section{COHORT DEFINITION AND FOLLOW UP}

The study cohort was defined as all German male workers of the five study plants who were: (a) actively employed on 1 January 1981 , or $(b)$ retired and alive on 1 January 1981. Men were not included into the cohort if they were either 85 years of age or older at the beginning of follow up or if they had been employed for less than one year in any one of the study plants.

The cohort was defined from 1 January 1981 for two practical reasons: firstly, in most states in Germany, legislation requires the storage of death certificates only for 10 years, after which time they may be destroyed; and secondly, 1981 was the first full year for which electronic data on potential cohort members were available from the insurance companies and the study plants. Accurate enumeration of the cohort before this date would have been logistically impossible because it would have required identifying potential cohort members through paper records within the extensive archives of the rubber companies.

Cohort eligibility was limited to those who had been employed for at least one year, because this restriction eliminates short term workers who might not have adequately long employment to accumulate meaningful exposure levels. Also more transient men might have exposures from many jobs and might behave differently with respect to their health from others employed for longer times in the rubber industry. ${ }^{38}$ Men aged 85 years or older were excluded from the cohort, because the reported causes of death on the death certificates of very old people may be less valid and the specific causes less likely to be occupationally related. ${ }^{39} 40$

Data on both women and non-German men meeting essentially the same cohort definitions are currently being collected from the same sources, and will be analysed and presented in a future report. Employees first hired after 1 January 1981 ( $\mathrm{n}=3.721$ ) were also followed up to facilitate future follow up efforts. However, the maximum period from the beginning of employment until the end of follow up (31 December 1991) was only 11 years for these workers. Only 13 deaths (20 expected) were observed among these workers and no man had died from cancer $(2 \cdot 8$ expected). New employees were therefore not included in this analysis of cancer mortality.

According to the cohort definition two discrete subcohorts were identified. The active workers represented a cross section of all those who were hired before and were still employed on 1 January 1981 . Of these, cohort members who retired during the follow up period remained in the active subcohort for analyses. This subcohort, although not an inception cohort, resembles a typically defined active working cohort, and will be followed up for mortality in the future. Retired employees consisted of a cross section of all retirees who were alive on 1 January 1981 and aged less than 85 years. This group was specifically included to enhance the study's ability to detect risks associated with earlier conditions in the industry and among older men, who are at greater risk of mortality for most causes. Most of the retirees were men who remained employed until reaching the usual age of retirement (60-65 years); however, the group also included men who retired early, as part of the efforts to reduce the workforce, or who retired from the workforce for reasons of health.

For most cohort members follow up started on 1 January 1981 and ended on 31 December 1991. For those who had not worked for at least one year before 1 January 1981, follow up began after the one year period of employment was completed. Person time observed was censored as men reached the age of 85 years. For those lost to follow up $(n=39)$, person time was accumulated until the last date on which the person was known to be alive.

\section{COHORT ENUMERATION AND EMPLOYMENT} HISTORY

Cohort members were identified through the computerised files of the health insurance companies and the personnel files of the rubber plants. For men with missing data elements, cohort eligibility was established with additional data from the rubber companies, such as personnel paper charts and other docu- 
ments. Work histories within the respective companies were obtained on $99.7 \%$ of the cohort members. Employer personnel files were used for active employees. For retirees and employees with missing information in the electronic files, work histories were abstracted from records. Work histories were reconstructed with cost centre accounting codes (Kostenstellen). These were originally generated for accounting purposes, but permit identification of employment in specific working areas and the respective time periods. Analyses of mortality by work area will be presented in future publications.

\section{VITAL STATUS AND ASSESSMENT OF CAUSE OF DEATH}

Health insurance data and personnel files of the participating plants were used to find the vital status of cohort members at the end of the observation period. Cohort members were assumed to be alive if they were either actively employed in one of the study plants or if they maintained insurance with the health insurance companies providing data on the cohort. Insurance claims were screened until the end of 1992 to identify any deaths occurring late in the follow up period, and for which reports might have been delayed. For all other cohort members (about 4500) vital status was ascertained through the appropriate local population registry by finding the last known address. All moves must be registered according to German law. If the cohort member was found to be dead the place of death was recorded. Overall ascertainment of vital status for the cohort was nearly complete $(99 \cdot 7 \%)$, with only 39 employees remaining with vital status unknown as of the end of the follow up period (table 1).

For all cohort members reported to have died ( $n=2719$ ), information from the population registry was used to request a copy of the death certificate from the respective community health department. Because death certificates were not readily available for research purposes in Germany until recently, a great effort was necessary to secure full cooperation of the health department officials to obtain all available death certificates of those who died. Death certificates were successfully obtained on $2631(96.8 \%)$ of the decedents.

The causes of death were identified through the death certificates. The underlying causes of death were coded according to the ninth revision of the international classification of diseases (ICD-9) by four professional nosolo-

Table 1 Assessment of vital status and cause of death at the end of follow up

\begin{tabular}{|c|c|c|c|}
\hline & Combined $(n=11663)$ & Retired $^{*}(n=4127)$ & $\operatorname{Active}^{*}(n=7536)$ \\
\hline & $n(\%)$ & $n(\%)$ & $n(\%)$ \\
\hline $\begin{array}{l}\text { Vital status: } \\
\text { Alive } \\
\text { Dead } \\
\text { Unknown }\end{array}$ & $\begin{array}{c}8905(76 \cdot 4) \\
2719(23 \cdot 3) \\
39(0 \cdot 3)\end{array}$ & $\begin{array}{c}2118(51 \cdot 3) \\
1992(48 \cdot 3) \\
17(0 \cdot 4)\end{array}$ & $\begin{array}{c}6787(90 \cdot 1) \\
727(9 \cdot 6) \\
22(0 \cdot 3)\end{array}$ \\
\hline $\begin{array}{l}\text { Cause of death: } \\
\text { Known } \\
\text { Unknown }\end{array}$ & $\begin{array}{c}2631(96 \cdot 8) \\
88(3 \cdot 2)\end{array}$ & $\begin{array}{c}1932(97 \cdot 0) \\
60(3.0)\end{array}$ & $\begin{array}{c}699(96 \cdot 1) \\
28(3 \cdot 9)\end{array}$ \\
\hline
\end{tabular}

^On 1 January 1981 . gists from the State Institute of Statistics of North-Rhine/Westphalia. These people were selected because of their experience with coding of death certificates, so that the study deaths would be coded in the same manner as the deaths from the general population, and therefore be comparable with the deaths on which mortalities for the general population are based.

\section{DATA ANALYSIS}

Mortality experience of the cohort was compared with the mortality of the general male population using standardised mortality ratio (SMR) analyses. The population of western Germany was used as the reference, because the participating plants were located in three different states of western Germany. The National Institute of Statistics provided data on the number of residents by calendar year, five year age group, nationality (German $v$ non-German) and sex, as well as the number of respective deaths by ICD-9 categories. The SMRs were standardised on calendar year and five year age groups. Person-years at risk were calculated for each cohort member and summed across all men in each analytical stratum of interest-for example, calendar year, age group, cohort status, year of hire, etc-by Epicure and programs written in SAS 6.10. The respective stratum-specific rates from the reference population of German men were multiplied by these person-year weights to obtain the expected number of deaths. The SMRs were calculated by dividing the observed number of deaths from specific causes by the expected number.

For causes with at least five expected deaths, approximate $95 \%$ CIs were constructed. ${ }^{41}$ For causes with less than five expected deaths, and for which the observed number of deaths was at least two, $95 \%$ CIs were constructed with an exact method. ${ }^{41}$ The 95\% CIs are not reported for causes with one or no observed death. Analyses were conducted for the total cohort and by employment status (active, retired) at the beginning of follow up. The mortality from cancer of specific sites was further stratified by year of hire and years since hire to assess possible associations with occupational factors.

\section{Results}

COHORT CHARACTERISTICS

The study cohort consisted of 11663 German men, who were retired $(n=4127)$ on 1 January 1981 , or who were actively employed $(n=7536)$ on 1 January 1981. Figure 1 shows that most retirees were over 60 years of age on 1 January 1981, whereas most of the active workers were 40 to 59 years old at the beginning of follow up. Figure 2 shows the distribution of cohort members by year of hire. The retirees are widely distributed over the early categories, with close to 500 who were hired before 1930, and only a few who started work after 1959. Most active workers had been hired by one of the study plants between 1950 and 1969. 
Figure 1 Distribution of cohort members $(n=$ $11663)$ by cohort status (retired, active) and by age at beginning of follow up.

Figure 2 Distribution of cohort members $(n=$ 11 663) by cohort status (retired, active) and by year of hire.
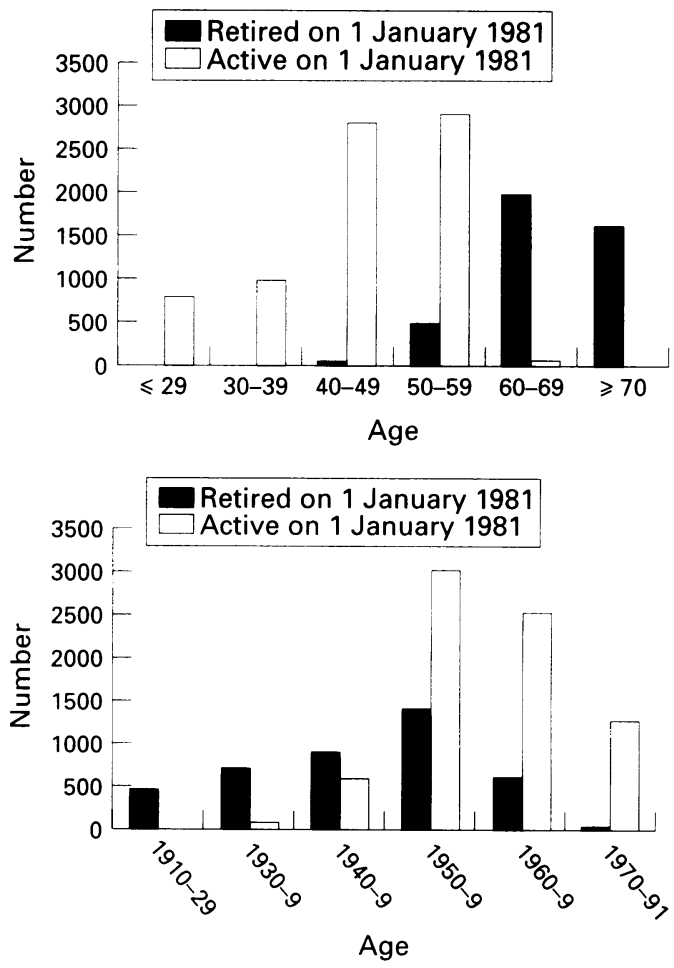

The study cohort generated a total of 110 512 person-years of follow up: 31260 from the retirees, and 79252 from the active workers. Table 2 shows the distribution of person-years of follow up by age at risk and years since hire. Retirees contributed very little time of follow up to the categories of less than 10 years $(0 \cdot 1 \%)$, and $10-19$ years $(4 \cdot 3 \%)$ since hire, whereas close to one third of the person years of active workers fell into the lower categories of years since hire: $<10$ years $(9 \cdot 5 \%)$ and $10-19$ years $(17 \cdot 7 \%)$.

TOTAL AND NON-CANCER MORTALITIES

The mortality from all causes in the study cohort during the follow up period, 1 January 1981 until 31 December 1991, was higher than expected from national rates (SMR 108; 95\% CI 104-112, table 3). This increased mortality, however, is explained by an excess of deaths among the subcohort of retirees (SMR 113; 95\% CI 108-118), whereas the active employees showed a slightly lower SMR of 95 (95\% CI 89-103) for all causes. Table 3 shows non-cancer causes of death, which are of interest in this paper mainly to better understand the cohort and their broader patterns of mortality. The retirees tended to slightly exceed expectation, and the active workers mostly fell below the expected number. One example where the contrast was strong is mortality from diabetes mellitus, for which a substantial excess was found among retirees (SMR 181; 95\% CI 131-244), but active workers experienced a relative deficit of deaths from diabetes (SMR 70;95\% CI 26-153).

Diseases of the circulatory system (ICD-9 390-459) show an increase among retirees (SMR 109; 95\% CI 102-116), whereas active workers have a small deficit (SMR 89; 95\% CI 79-101). The excess among retirees cannot be explained completely by increases in one single subcategory of circulatory diseases. Ischaemic heart disease, which accounts for the greatest number of deaths from diseases of the circulatory system, is slightly increased among retirees (SMR 103; 95\% CI 94-113), and significantly lower among active workers (SMR 83; 95\% CI 70-98). A substantial excess in the mortality from hypertensive diseases $(n=39)$ is found among retirees (SMR 202; 95\% CI 143-276), and although based on only seven deaths the mortality is also increased among active workers (SMR 125; 95\% CI 50-258).

Deaths from chronic obstructive pulmonary diseases (ICD-9 490-496) were identified more often than expected (SMR 115; 95\% CI 96-135). The excess, however, was found only among retirees (SMR 122; 95\% CI 101-146); mortality among the subcohort of active workers lay moderately below expectation (SMR 85; 95\% CI 52-131). The largest deficit among all non-cancer causes was found for infectious and parasitic diseases (SMR 26; 95\% CI 8-61).

\section{CANCER MORTALITY}

The study cohort experienced a significantly increased mortality from all cancers (SMR $111 ; 95 \%$ CI 103-119, table 3). Excess deaths from cancer occurred both among retirees (SMR 114; 95\% CI 104-124) and active workers (SMR 106; 95\% CI 93-119). A significant increase was identified for the mortality from cancer of the lung (SMR 130;95\% CI 115-147) and pleura (SMR 401;95\% CI 234-642). Increased mortalities, with SMRs higher than 100 but with CIs including 100, were found for cancers of the pharynx (SMR 144; 95\% CI 76-246), oesophagus (SMR

Table 2 Distribution of person-years and deaths by age at risk, cohort status, and years since hire

\begin{tabular}{|c|c|c|c|c|c|c|c|c|c|c|c|c|c|c|c|c|}
\hline \multirow[b]{3}{*}{ Age at risk } & \multicolumn{8}{|c|}{ Retired ${ }^{\star}$ (years since hire) } & \multicolumn{8}{|c|}{ Active $^{\star}$ (years since hire) } \\
\hline & \multicolumn{2}{|c|}{$<10$} & \multicolumn{2}{|c|}{$10-19$} & \multicolumn{2}{|l|}{$\geqslant 20$} & \multicolumn{2}{|l|}{ total† } & \multicolumn{2}{|l|}{$<10$} & \multicolumn{2}{|l|}{$10-19$} & \multicolumn{2}{|l|}{$\geqslant 20$} & \multicolumn{2}{|l|}{ total } \\
\hline & py & deaths & py & deaths & py & deaths & py & deaths & py & deaths & py & deaths & py & deaths & py & deaths \\
\hline$\leqslant 29$ & 6 & 0 & 7 & 0 & 0 & 0 & 13 & 0 & 4092 & 1 & 1180 & 0 & 0 & 0 & 5272 & 1 \\
\hline $30-39$ & 0 & 0 & 30 & 1 & 2 & 0 & 32 & 1 & 1894 & 3 & 3984 & 4 & 1002 & 1 & 6880 & 8 \\
\hline $40-49$ & 0 & 0 & 52 & 0 & 152 & 2 & 204 & 2 & 1031 & 5 & 4869 & 24 & 14011 & 61 & 19911 & 90 \\
\hline $50-59$ & 8 & 1 & 133 & 5 & 1163 & 64 & 1305 & 70 & 470 & 6 & 3379 & 36 & 28737 & 293 & 32586 & 335 \\
\hline 60-69 & 20 & 0 & 685 & 23 & 10882 & 382 & 11587 & 405 & 53 & 0 & 613 & 8 & 13774 & 283 & 14439 & 291 \\
\hline 70-79 & 1 & 0 & 438 & 27 & 14618 & 1055 & 15056 & 1082 & 0 & 0 & 5 & 0 & 158 & 2 & 163 & 2 \\
\hline$\geqslant 80$ & 0 & 0 & 12 & 1 & 3052 & 415 & 3064 & 416 & 0 & 0 & 0 & 0 & 0 & 0 & 0 & 0 \\
\hline Total & 35 & 1 & 1356 & 57 & 29869 & 1918 & 31260 & 1976 & 7540 & 15 & 14031 & 72 & 57681 & 640 & 79252 & 727 \\
\hline
\end{tabular}

*On 1 January 1981

†For 16 deceased retirees date of hire was unknown.

py $=$ person-years. 
Table 3 Number of observed deaths and SMRs with 95\% CIs among male German rubber workers ( $n=11663)$ between 1981 and 1991 by cause of death and cohort status

\begin{tabular}{|c|c|c|c|c|c|c|c|c|c|}
\hline \multirow[b]{2}{*}{ Cause of death } & \multirow[b]{2}{*}{$I C D-9$} & \multicolumn{3}{|c|}{ Combined $(n=11663)$} & \multicolumn{2}{|c|}{ Retired $^{\star}(n=4127)$} & \multicolumn{3}{|c|}{ Active $^{*}(n=7536)$} \\
\hline & & Obs & SMR† & $(95 \% C I)$ & Obs SMR† & $(95 \% C I)$ & Obs & SMR† & $(95 \% C I)$ \\
\hline $\begin{array}{l}\text { All causes: } \\
\text { Infectious and parasitic diseases } \\
\text { All cancers: } \\
\text { Cancer of: }\end{array}$ & $\begin{array}{l}000-999 \\
000-139 \\
140-208\end{array}$ & $\begin{array}{r}2719 \\
5 \\
773\end{array}$ & $\begin{array}{r}108 \\
26 \\
111\end{array}$ & $\begin{array}{l}(104-112) \\
(8-61) \\
(103-119)\end{array}$ & $\begin{array}{rr}1992 & 113 \\
4 & 34 \\
520 & 114\end{array}$ & $\begin{array}{l}(108-118) \\
(9-88) \\
(104-124)\end{array}$ & $\begin{array}{r}727 \\
1 \\
253\end{array}$ & $\begin{array}{r}95 \\
13 \\
106\end{array}$ & $\frac{(89-103)}{(93-119)}$ \\
\hline $\begin{array}{l}\text { Cancer or: } \\
\text { Lip or oral cavity or pharynx } \\
\text { Pharynx } \\
\text { Digestive organs or peritoneum } \\
\text { Oesophagus } \\
\text { Stomach } \\
\text { Colon } \\
\text { Rectum } \\
\text { Liver or gallbladder } \\
\text { Pancreas } \\
\text { Respiratory and intrathoracic }\end{array}$ & $\begin{array}{l}140-149 \\
146-149 \\
150-159 \\
150 \\
151 \\
153 \\
154 \\
155-156 \\
157\end{array}$ & $\begin{array}{r}24 \\
13 \\
223 \\
21 \\
72 \\
47 \\
36 \\
15 \\
23\end{array}$ & $\begin{array}{r}125 \\
144 \\
96 \\
120 \\
110 \\
84 \\
123 \\
59 \\
71\end{array}$ & $\begin{array}{l}(80-186) \\
(76-246) \\
(84-109) \\
(74-183) \\
(86-139) \\
(62-112) \\
(86-170) \\
(33-98) \\
(45-106)\end{array}$ & $\begin{array}{rr}13 & 193 \\
7 & 244 \\
144 & 92 \\
10 & 121 \\
44 & 95 \\
34 & 87 \\
24 & 118 \\
9 & 54 \\
16 & 79\end{array}$ & $\begin{array}{l}(103-331) \\
(98-502) \\
(78-109) \\
(58-223) \\
(69-127) \\
(61-122) \\
(76-176) \\
(24-102) \\
(45-128)\end{array}$ & $\begin{array}{r}11 \\
6 \\
79 \\
11 \\
28 \\
13 \\
12 \\
6 \\
7\end{array}$ & $\begin{array}{r}88 \\
97 \\
103 \\
119 \\
148 \\
77 \\
135 \\
70 \\
58\end{array}$ & $\begin{array}{l}(44-158) \\
(36-212) \\
(82-129) \\
(59-212) \\
(99-214) \\
(41-132) \\
(69-235) \\
(26-153) \\
(23-119)\end{array}$ \\
\hline $\begin{array}{l}\text { organs } \\
\text { Larynx } \\
\text { Trachea or bronchus or lung } \\
\text { Pleura } \\
\text { Skin } \\
\text { Male genitourinary organs } \\
\text { Prostate } \\
\text { Bladder } \\
\text { Brain or central nervous system } \\
\text { Without specification of site } \\
\text { Lymphatic and haematopoietic }\end{array}$ & $\begin{array}{l}160-165 \\
161 \\
162 \\
163 \\
172-173 \\
185-189 \\
185 \\
188 \\
191-192 \\
199\end{array}$ & $\begin{array}{r}287 \\
13 \\
257 \\
17 \\
2 \\
128 \\
71 \\
35 \\
9 \\
51\end{array}$ & $\begin{array}{r}135 \\
129 \\
130 \\
401 \\
29 \\
107 \\
108 \\
124 \\
83 \\
130\end{array}$ & $\begin{array}{l}(119-151) \\
(69-221) \\
(115-147) \\
(234-642) \\
(3-105) \\
(89-127) \\
(84-136) \\
(86-172) \\
(38-158) \\
(97-172)\end{array}$ & $\begin{array}{rr}184 & 142 \\
8 & 157 \\
163 & 134 \\
13 & 533 \\
1 & 28 \\
106 & 112 \\
63 & 110 \\
28 & 126 \\
4 & 91 \\
37 & 140\end{array}$ & $\begin{array}{l}(122-164) \\
(67-308) \\
(114-156) \\
(284-912) \\
-(92-136) \\
(85-141) \\
(84-183) \\
(25-232) \\
(99-193)\end{array}$ & $\begin{array}{r}103 \\
5 \\
94 \\
4 \\
1 \\
22 \\
8 \\
7 \\
5 \\
14\end{array}$ & $\begin{array}{r}124 \\
101 \\
124 \\
222 \\
30 \\
87 \\
92 \\
113 \\
78 \\
110\end{array}$ & $\begin{array}{l}(101-150) \\
(33-236) \\
(100-152) \\
(61-570) \\
-(54-131) \\
(40-182) \\
(45-234) \\
(25-182) \\
(60-185)\end{array}$ \\
\hline $\begin{array}{l}\text { tissue } \\
\text { Lymphatic system } \\
\text { Leukaemia } \\
\text { Other neoplasms } \\
\text { Diabetes mellitus } \\
\text { Diseases of the circulatory system } \\
\text { Hypertensive disease } \\
\text { Ischaemic heart disease } \\
\text { Cerebrovascular disease } \\
\text { Diseases of the respiratory system } \\
\text { Chronic obstructive pulmonary }\end{array}$ & $\begin{array}{l}200-208 \\
200-203 \\
204-208 \\
210-239 \\
250 \\
390-459 \\
401-405 \\
410-414 \\
430-438 \\
460-519\end{array}$ & $\begin{array}{r}46 \\
17 \\
29 \\
8 \\
49 \\
1237 \\
46 \\
580 \\
238 \\
174\end{array}$ & $\begin{array}{r}112 \\
78 \\
148 \\
39 \\
152 \\
104 \\
184 \\
97 \\
96 \\
97\end{array}$ & $\begin{array}{l}(82-149) \\
(46-126) \\
(99-213) \\
(17-77) \\
(112-201) \\
(99-110) \\
(135-246) \\
(90-106) \\
(84-109) \\
(83-113)\end{array}$ & $\begin{array}{rr}29 & 109 \\
7 & 53 \\
22 & 164 \\
5 & 35 \\
43 & 181 \\
980 & 109 \\
39 & 202 \\
439 & 103 \\
195 & 93 \\
150 & 103\end{array}$ & $\begin{array}{l}(73-157) \\
(21-109) \\
(103-249) \\
(11-82) \\
(131-244) \\
(102-116) \\
(143-276) \\
(94-113) \\
(81-107) \\
(87-121)\end{array}$ & $\begin{array}{r}17 \\
10 \\
7 \\
3 \\
6 \\
257 \\
7 \\
141 \\
43 \\
24\end{array}$ & $\begin{array}{r}116 \\
118 \\
113 \\
49 \\
70 \\
89 \\
125 \\
83 \\
108 \\
72\end{array}$ & $\begin{array}{l}(67-186) \\
(57-217) \\
(45-232) \\
(10-142) \\
(26-153) \\
(79-101) \\
(50-258) \\
(70-98) \\
(78-146) \\
(46-108)\end{array}$ \\
\hline $\begin{array}{l}\text { disease } \\
\text { Diseases of the digestive system } \\
\text { Chronic liver disease } \\
\text { Diseases of the genitourinary }\end{array}$ & $\begin{array}{l}490-496 \\
520-579 \\
571\end{array}$ & $\begin{array}{r}139 \\
125 \\
93\end{array}$ & $\begin{array}{r}115 \\
90 \\
117\end{array}$ & $\begin{array}{l}(96-135) \\
(75-107) \\
(95-144)\end{array}$ & $\begin{array}{rr}119 & 122 \\
67 & 87 \\
43 & 124\end{array}$ & $\begin{array}{l}(101-146) \\
(67-110) \\
(90-167)\end{array}$ & $\begin{array}{l}20 \\
58 \\
50\end{array}$ & $\begin{array}{r}85 \\
94 \\
112\end{array}$ & $\begin{array}{l}(52-131) \\
(71-121) \\
(83-148)\end{array}$ \\
\hline $\begin{array}{l}\text { system } \\
\text { Injury and poisoning }\end{array}$ & $\begin{array}{l}580-608 \\
800-999\end{array}$ & $\begin{array}{l}22 \\
94\end{array}$ & $\begin{array}{l}79 \\
89\end{array}$ & $\begin{array}{l}(50-120) \\
(72-109)\end{array}$ & $\begin{array}{rr}18 & 81 \\
50 & 111\end{array}$ & $\begin{array}{l}(48-128) \\
(82-146)\end{array}$ & $\begin{array}{r}4 \\
44\end{array}$ & $\begin{array}{l}72 \\
73\end{array}$ & $\begin{array}{l}(19-184) \\
(53-98)\end{array}$ \\
\hline
\end{tabular}

*On 1 January 1981.

†SMR based on the male population of western Germany as reference.

120; 95\% CI 74-183), stomach (SMR 110; 95\% CI 86-139), rectum (SMR 123; 95\% CI 86-170), larynx (SMR 129; 95\% CI 69-221), prostate (SMR 108; 95\% CI 84-136), and the bladder (SMR 124; 95\% CI 86-172), as well as for leukaemia (SMR 148; 95\% CI 99-213). The excess deaths from pharyngeal and laryngeal cancer occurred among the subcohort of retirees-that is, those who were already retired at the beginning of follow up, whereas the mortality from stomach cancer was increased only among the subcohort of active workers. To decide whether any of these findings are possibly related to workplace hazards, further stratified analyses were performed for the total cohort and by cohort status at the beginning of follow up.

Year of hire

Table 4 shows the SMRs for a subset of cancer sites, chosen on the basis of findings from previously published studies, stratified by year of hire. Lung cancer, with by far the largest number of observed deaths from cancer $(\mathbf{n}=$ 257), had consistently increased SMRs for all strata. The highest excess was found among retirees hired before 1950 (SMR 148; 95\% CI 119-182), but the mortality was also increased among all cohort members hired since 1960 (SMR 136; 95\% CI 104-175). The strongest association with a cancer site, however, was found for deaths from pleural cancer $(n=17)$ with significantly increased mortality among workers hired before 1950 (SMR 630; 95\% CI 289-1,197), and between 1950 and 1959 (SMR 341; 95\% CI 125-743).

Mortality from laryngeal cancer seemed to be increased for those hired before 1950 as well as those hired since 1960; stratified by cohort status, the increase among retirees is only found among those hired before 1960, whereas mortality among active workers is increased among those hired since 1960 (SMR $274 ; 95 \%$ CI 89-638). The number of observed events $(n=13)$, however, is quite small. Mortality from pharyngeal cancer, with the same number of observed cases, showed a similar pattern, with an increased mortality among retirees hired before 1960 , whereas the increased risk among active employees was found among workers hired since 1960 (SMR 198; 95\% CI 64-462).

The SMRs that were above 100 were for oesophageal and rectal cancer in cohort members with earlier years of hire $(<1960)$, regardless of cohort status. In contrast, the mortality from stomach cancer was increased among workers hired since 1960 . The risk estimates for active workers were increased across all categories of year of hire. A significantly higher mortality from bladder cancer was found in workers hired since 1960 (SMR 214; 95\% CI 107-384). This excess occurred similarly among active and retired workers. There was also a slight excess of deaths among retirees (just over 10\%) hired before 1960 . 
Table 4 Number of observed deaths and SMRs with 95\% CIs among male German rubber workers ( $n=11663)$ between 1981 and 1991 by cancer site, cohort status, and year of hire

\begin{tabular}{|c|c|c|c|c|c|c|c|c|c|c|}
\hline \multirow{2}{*}{$\begin{array}{l}\text { Cancer site } \\
(I C D-9)\end{array}$} & \multirow{2}{*}{$\begin{array}{l}\text { Cohort } \\
\text { status* }\end{array}$} & \multicolumn{3}{|c|}{ Year of hire $<1950$} & \multicolumn{3}{|c|}{ Year of hire $1950-9$} & \multicolumn{3}{|c|}{ Year of hire $\geqslant 1960$} \\
\hline & & Obs & SMR† & $(95 \% C I)$ & Obs & SMR† & $(95 \% C I)$ & Obs & SMR† & $(95 \% C I)$ \\
\hline $\begin{array}{l}\text { All } \\
(140-208)\end{array}$ & $\begin{array}{l}\text { combined } \\
\text { retired } \\
\text { active }\end{array}$ & $\begin{array}{r}314 \\
275 \\
39\end{array}$ & $\begin{array}{l}118 \\
119 \\
110\end{array}$ & $\begin{array}{l}(105-131) \\
(105-134) \\
(78-150)\end{array}$ & $\begin{array}{l}282 \\
168 \\
114\end{array}$ & $\begin{array}{r}103 \\
111 \\
94\end{array}$ & $\begin{array}{l}(92-116) \\
(95-129) \\
(77-113)\end{array}$ & $\begin{array}{r}173 \\
73 \\
100\end{array}$ & $\begin{array}{l}114 \\
106 \\
121\end{array}$ & $\begin{array}{l}(98-132) \\
(83-133) \\
(98-147)\end{array}$ \\
\hline $\begin{array}{l}\text { Pharynx } \\
(146-149)\end{array}$ & $\begin{array}{l}\text { combined } \\
\text { retired } \\
\text { active }\end{array}$ & $\begin{array}{l}3 \\
3 \\
0\end{array}$ & $\begin{array}{l}154 \\
232 \\
-\end{array}$ & $\begin{array}{l}(32-450) \\
(48-678) \\
-\end{array}$ & $\begin{array}{l}4 \\
3 \\
1\end{array}$ & $\begin{array}{r}98 \\
279 \\
33\end{array}$ & $\begin{array}{l}(27-252) \\
(58-815) \\
-\end{array}$ & $\begin{array}{l}6 \\
1 \\
5\end{array}$ & $\begin{array}{l}200 \\
207 \\
198\end{array}$ & $\frac{(73-435)}{(64-462)}$ \\
\hline $\begin{array}{l}\text { Oesophagus } \\
(150)\end{array}$ & $\begin{array}{l}\text { combined } \\
\text { retired } \\
\text { active }\end{array}$ & $\begin{array}{l}8 \\
6 \\
2\end{array}$ & $\begin{array}{l}156 \\
151 \\
170\end{array}$ & $\begin{array}{l}(67-307) \\
(56-330) \\
(21-614)\end{array}$ & $\begin{array}{l}9 \\
3 \\
6\end{array}$ & $\begin{array}{l}119 \\
104 \\
129\end{array}$ & $\begin{array}{l}(54-226) \\
(21-303) \\
(47-281)\end{array}$ & $\begin{array}{l}4 \\
1 \\
3\end{array}$ & $\begin{array}{l}84 \\
77 \\
87\end{array}$ & $\frac{(23-216)}{(18-255)}$ \\
\hline $\begin{array}{l}\text { Stomach } \\
(151)\end{array}$ & $\begin{array}{l}\text { combined } \\
\text { retired } \\
\text { active }\end{array}$ & $\begin{array}{r}27 \\
22 \\
5\end{array}$ & $\begin{array}{r}100 \\
91 \\
171\end{array}$ & $\begin{array}{l}(66-145) \\
(57-138) \\
(55-399)\end{array}$ & $\begin{array}{l}25 \\
13 \\
12\end{array}$ & $\begin{array}{r}102 \\
87 \\
125\end{array}$ & $\begin{array}{l}(66-150) \\
(46-149) \\
(64-218)\end{array}$ & $\begin{array}{r}19 \\
8 \\
11\end{array}$ & $\begin{array}{l}144 \\
117 \\
174\end{array}$ & $\begin{array}{l}(87-225) \\
(50-230) \\
(87-311)\end{array}$ \\
\hline $\begin{array}{l}\text { Colon } \\
(153)\end{array}$ & $\begin{array}{l}\text { combined } \\
\text { retired } \\
\text { active }\end{array}$ & $\begin{array}{r}26 \\
22 \\
4\end{array}$ & $\begin{array}{l}115 \\
109 \\
155\end{array}$ & $\begin{array}{l}(75-168) \\
(69-166) \\
(42-396)\end{array}$ & $\begin{array}{r}13 \\
8 \\
5\end{array}$ & $\begin{array}{l}61 \\
63 \\
58\end{array}$ & $\begin{array}{l}(33-105) \\
(27-125) \\
(19-136)\end{array}$ & $\begin{array}{l}8 \\
4 \\
4\end{array}$ & $\begin{array}{l}70 \\
69 \\
71\end{array}$ & $\begin{array}{l}(30-138) \\
(19-177) \\
(19-182)\end{array}$ \\
\hline $\begin{array}{l}\text { Rectum } \\
\text { (154) }\end{array}$ & $\begin{array}{l}\text { combined } \\
\text { retired } \\
\text { active }\end{array}$ & $\begin{array}{r}17 \\
14 \\
3\end{array}$ & $\begin{array}{l}142 \\
132 \\
226\end{array}$ & $\begin{array}{l}(83-228) \\
(72-221) \\
(47-661)\end{array}$ & $\begin{array}{r}14 \\
8 \\
6\end{array}$ & $\begin{array}{l}126 \\
123 \\
132\end{array}$ & $\begin{array}{l}(69-212) \\
(53-242) \\
(48-287)\end{array}$ & $\begin{array}{l}5 \\
2 \\
3\end{array}$ & $\begin{array}{l}83 \\
67 \\
99\end{array}$ & $\begin{array}{l}(27-193) \\
(8-241) \\
(20-288)\end{array}$ \\
\hline $\begin{array}{l}\text { Liver or gallbladder } \\
(155-156)\end{array}$ & $\begin{array}{l}\text { combined } \\
\text { retired } \\
\text { active }\end{array}$ & $\begin{array}{l}5 \\
3 \\
2\end{array}$ & $\begin{array}{r}52 \\
36 \\
146\end{array}$ & $\begin{array}{l}(17-120) \\
(7-105) \\
(18-528)\end{array}$ & $\begin{array}{l}4 \\
2 \\
2\end{array}$ & $\begin{array}{l}40 \\
35 \\
46\end{array}$ & $\begin{array}{l}(11-101) \\
(4-126) \\
(6-165)\end{array}$ & $\begin{array}{l}5 \\
3 \\
2\end{array}$ & $\begin{array}{r}93 \\
116 \\
72\end{array}$ & $\begin{array}{l}(30-218) \\
(24-339) \\
(9-260)\end{array}$ \\
\hline $\begin{array}{l}\text { Pancreas } \\
(157)\end{array}$ & $\begin{array}{l}\text { combined } \\
\text { retired } \\
\text { active }\end{array}$ & $\begin{array}{l}7 \\
5 \\
2\end{array}$ & $\begin{array}{r}59 \\
50 \\
110\end{array}$ & $\begin{array}{l}(24-122) \\
(16-116) \\
(13-396)\end{array}$ & $\begin{array}{r}10 \\
8 \\
2\end{array}$ & $\begin{array}{r}76 \\
115 \\
32\end{array}$ & $\begin{array}{l}(36-140) \\
(49-226) \\
(4-116)\end{array}$ & $\begin{array}{l}5 \\
2 \\
3\end{array}$ & $\begin{array}{l}69 \\
64 \\
73\end{array}$ & $\begin{array}{l}(22-160) \\
(8-229) \\
(15-212)\end{array}$ \\
\hline $\begin{array}{l}\text { Larynx } \\
(161)\end{array}$ & $\begin{array}{l}\text { combined } \\
\text { retired } \\
\text { active }\end{array}$ & $\begin{array}{l}5 \\
5 \\
0\end{array}$ & $\begin{array}{l}159 \\
199 \\
-\end{array}$ & $\begin{array}{l}(51-370) \\
(64-463) \\
-\end{array}$ & $\begin{array}{l}3 \\
3 \\
0\end{array}$ & $\begin{array}{r}71 \\
171 \\
-\end{array}$ & $\begin{array}{l}(15-208) \\
(35-499) \\
-\end{array}$ & $\begin{array}{l}5 \\
0 \\
5\end{array}$ & $\frac{191}{274}$ & $\frac{(62-444)}{(89-638)}$ \\
\hline $\begin{array}{l}\text { Trachea or bronchus } \\
\text { or lung } \\
\text { (162) }\end{array}$ & $\begin{array}{l}\text { combined } \\
\text { retired } \\
\text { active }\end{array}$ & $\begin{array}{r}103 \\
88 \\
15\end{array}$ & $\begin{array}{l}145 \\
148 \\
128\end{array}$ & $\begin{array}{l}(118-176) \\
(119-182) \\
(72-212)\end{array}$ & $\begin{array}{l}94 \\
50 \\
44\end{array}$ & $\begin{array}{l}116 \\
119 \\
113\end{array}$ & $\begin{array}{l}(94-142) \\
(88-157) \\
(82-151)\end{array}$ & $\begin{array}{l}60 \\
25 \\
35\end{array}$ & $\begin{array}{l}136 \\
132 \\
139\end{array}$ & $\begin{array}{l}(104-175) \\
(85-195) \\
(97-194)\end{array}$ \\
\hline $\begin{array}{l}\text { Pleura } \\
(163)\end{array}$ & $\begin{array}{l}\text { combined } \\
\text { retired } \\
\text { active }\end{array}$ & $\begin{array}{l}9 \\
7 \\
2\end{array}$ & $\begin{array}{l}630 \\
591 \\
820\end{array}$ & $\begin{array}{l}(289-1197) \\
(237-1217) \\
(99-2961)\end{array}$ & $\begin{array}{l}6 \\
5 \\
1\end{array}$ & $\begin{array}{l}341 \\
588 \\
110\end{array}$ & $\begin{array}{l}(125-743) \\
(191-1370) \\
-\end{array}$ & $\begin{array}{l}2 \\
1 \\
1\end{array}$ & $\begin{array}{l}195 \\
261 \\
155\end{array}$ & $\begin{array}{l}(24-704) \\
-\end{array}$ \\
\hline $\begin{array}{l}\text { Prostate } \\
(185)\end{array}$ & $\begin{array}{l}\text { combined } \\
\text { retired } \\
\text { active }\end{array}$ & $\begin{array}{r}42 \\
41 \\
1\end{array}$ & $\begin{array}{r}128 \\
131 \\
63\end{array}$ & $\begin{array}{l}(92-173) \\
(94-178) \\
-\end{array}$ & $\begin{array}{r}18 \\
12 \\
6\end{array}$ & $\begin{array}{r}82 \\
69 \\
133\end{array}$ & $\begin{array}{l}(49-130) \\
(36-121) \\
(49-289)\end{array}$ & $\begin{array}{r}10 \\
9 \\
1\end{array}$ & $\begin{array}{r}94 \\
111 \\
39\end{array}$ & $\begin{array}{l}(45-173) \\
(51-212) \\
-\end{array}$ \\
\hline $\begin{array}{l}\text { Bladder } \\
\text { (188) }\end{array}$ & $\begin{array}{l}\text { combined } \\
\text { retired } \\
\text { active }\end{array}$ & $\begin{array}{r}14 \\
13 \\
1\end{array}$ & $\begin{array}{r}110 \\
111 \\
93\end{array}$ & $\begin{array}{l}(60-184) \\
(59-190) \\
-\end{array}$ & $\begin{array}{r}10 \\
8 \\
2\end{array}$ & $\begin{array}{r}98 \\
114 \\
62\end{array}$ & $\begin{array}{l}(47-180) \\
(49-224) \\
(8-226)\end{array}$ & $\begin{array}{r}11 \\
7 \\
4\end{array}$ & $\begin{array}{l}214 \\
216 \\
211\end{array}$ & $\begin{array}{l}(107-384) \\
(87-446) \\
(57-541)\end{array}$ \\
\hline $\begin{array}{l}\text { Brain or central } \\
\text { nervous system } \\
(191-192)\end{array}$ & $\begin{array}{l}\text { combined } \\
\text { retired } \\
\text { active }\end{array}$ & $\begin{array}{l}3 \\
3 \\
0\end{array}$ & $\begin{array}{l}110 \\
156 \\
-\end{array}$ & $\begin{array}{l}(23-322) \\
(32-456) \\
-\end{array}$ & $\begin{array}{l}4 \\
1 \\
3\end{array}$ & $\begin{array}{l}83 \\
59 \\
97\end{array}$ & 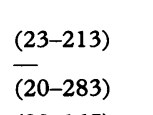 & $\begin{array}{l}2 \\
0 \\
2 \\
\end{array}$ & $\begin{array}{c}61 \\
-79\end{array}$ & $\frac{(7-220)}{(10-286)}$ \\
\hline $\begin{array}{l}\text { Lymphatic system } \\
(200-203)\end{array}$ & $\begin{array}{l}\text { combined } \\
\text { retired } \\
\text { active }\end{array}$ & $\begin{array}{l}3 \\
3 \\
0\end{array}$ & $\begin{array}{r}39 \\
47 \\
-\end{array}$ & $\begin{array}{l}(8-115) \\
(9-136) \\
-\end{array}$ & $\begin{array}{l}7 \\
3 \\
4\end{array}$ & $\begin{array}{l}80 \\
65 \\
96\end{array}$ & $\begin{array}{l}(32-165) \\
(13-191) \\
(26-246)\end{array}$ & $\begin{array}{l}7 \\
1 \\
6\end{array}$ & $\begin{array}{r}135 \\
48 \\
191\end{array}$ & $\frac{(54-277)}{(70-417)}$ \\
\hline $\begin{array}{l}\text { Leukaemia } \\
(204-208)\end{array}$ & $\begin{array}{l}\text { combined } \\
\text { retired } \\
\text { active }\end{array}$ & $\begin{array}{l}8 \\
8 \\
0\end{array}$ & $\begin{array}{l}103 \\
117 \\
-\end{array}$ & $\begin{array}{l}(45-204) \\
(50-230) \\
-\end{array}$ & $\begin{array}{r}17 \\
12 \\
5\end{array}$ & $\begin{array}{l}229 \\
273 \\
165\end{array}$ & $\begin{array}{l}(133-366) \\
(141-477) \\
(54-385)\end{array}$ & $\begin{array}{l}4 \\
2 \\
2\end{array}$ & $\begin{array}{r}93 \\
100 \\
87\end{array}$ & $\begin{array}{l}(25-238) \\
(12-359) \\
(11-314)\end{array}$ \\
\hline
\end{tabular}

*On 1 January 1981

tSMR based on the male population of western Germany as reference.

Leukaemia was the only investigated cancer for which the subgroup first employed between 1950 and 1959 was at highest risk (SMR 229; 95\% CI 133-366).

YEARS SINCE HIRE

The analysis by years since hire was performed to account for the latency period between the beginning of exposure and the observed deaths from cancer. For most occupationally related solid cancers, an empirical induction period of at least 10 years would be expected. Two deaths $(0.9$ expected) from lung cancer were identified after less than 10 years since hire. However, mortality from lung cancer was also increased after 10-19 years (SMR 165; 95\% CI 96-265) and after 20 or more years (SMR 129; $95 \%$ CI 113-146). Deaths from pleural cancer were not identified during the first 20 years since hire. A striking excess occurred after 20 and more years among the retired (SMR 555; 95\% CI 295-950) and active workers (SMR 249; 95\% CI 68-637).
Although one worker had died from laryngeal cancer less than 10 years after hire, mortality from cancer of the larynx was also increased after 20 and more years since hire (SMR 130; 95\% CI 67-226) and mostly affected those who were retired at the beginning of follow up.

Deaths from pharyngeal cancer were identified only after more than 10 years since hire, and there was a significant excess in mortality among retirees after 20 and more years (SMR 255; 95\% CI 102-526). Among active workers, there was an increased mortality from stomach cancer 10-19 years (SMR 235; 95\% CI 64-602), and 20 and more years since hire (SMR 142; 95\% CI 91-211).

A notable increase in mortality after 10-19 years since hire was found for cancers of the bladder (SMR 281; 95\% CI 58-820) and the lymphatic system (SMR 251; 95\% CI 52-733). However, the numbers of observed deaths were small (three deaths for each type of cancer) and, accordingly, the CIs were large. For bladder cancer there was also a 
Table 5 Number of observed cancer deaths and (SMRs) with 95\% CIs among male German rubber workers ( $n=11663$ ) between 1981 and 1991 by cancer site, cohort status, and years since hire

\begin{tabular}{|c|c|c|c|c|c|c|c|c|c|c|}
\hline \multirow{2}{*}{$\begin{array}{l}\text { Cancer site } \\
\text { (ICD-9) }\end{array}$} & \multirow{2}{*}{$\begin{array}{l}\text { Cohort } \\
\text { status* }\end{array}$} & \multicolumn{3}{|c|}{$<10$ years since hire } & \multicolumn{3}{|c|}{ 10-19 years since hire } & \multicolumn{3}{|c|}{$\geqslant 20$ years since hire } \\
\hline & & Obs & $S M R$ & $(95 \% C I)$ & Obs & SMR† & $(95 \% C I)$ & Obs & SMR† & $(95 \% C I)$ \\
\hline $\begin{array}{l}\text { All } \\
(140-208)\end{array}$ & $\begin{array}{l}\text { combined } \\
\text { retired } \\
\text { active }\end{array}$ & $\begin{array}{l}3 \\
0 \\
3\end{array}$ & $\begin{array}{r}79 \\
0 \\
83\end{array}$ & $\frac{(16-229)}{(17-242)}$ & $\begin{array}{l}42 \\
16 \\
26\end{array}$ & $\begin{array}{l}120 \\
114 \\
124\end{array}$ & $\begin{array}{l}(86-162) \\
(65-185) \\
(81-181)\end{array}$ & $\begin{array}{l}724 \\
500 \\
224\end{array}$ & $\begin{array}{l}111 \\
114 \\
104\end{array}$ & $\begin{array}{l}(103-119) \\
(104-125) \\
(91-119)\end{array}$ \\
\hline $\begin{array}{l}\text { Pharynx } \\
(146-149)\end{array}$ & $\begin{array}{l}\text { combined } \\
\text { retired } \\
\text { active }\end{array}$ & $\begin{array}{l}0 \\
0 \\
0\end{array}$ & $\begin{array}{l}0 \\
0 \\
0\end{array}$ & $\begin{array}{l}- \\
-\end{array}$ & $\begin{array}{l}1 \\
0 \\
1\end{array}$ & $\begin{array}{r}131 \\
0 \\
153\end{array}$ & $\begin{array}{l}- \\
-\end{array}$ & $\begin{array}{r}12 \\
7 \\
5\end{array}$ & $\begin{array}{r}148 \\
255 \\
93\end{array}$ & $\begin{array}{l}(76-258) \\
(102-526) \\
(30-216)\end{array}$ \\
\hline $\begin{array}{l}\text { Oesophagus } \\
(150)\end{array}$ & $\begin{array}{l}\text { combined } \\
\text { retired } \\
\text { active }\end{array}$ & $\begin{array}{l}0 \\
0 \\
0\end{array}$ & $\begin{array}{l}0 \\
0 \\
0\end{array}$ & E & $\begin{array}{l}1 \\
0 \\
1\end{array}$ & $\begin{array}{r}87 \\
0 \\
116\end{array}$ & E & $\begin{array}{l}20 \\
10 \\
10\end{array}$ & $\begin{array}{l}124 \\
127 \\
121\end{array}$ & $\begin{array}{l}(76-191) \\
(61-234) \\
(58-222)\end{array}$ \\
\hline $\begin{array}{l}\text { Stomach } \\
(151)\end{array}$ & $\begin{array}{l}\text { combined } \\
\text { retired } \\
\text { active }\end{array}$ & $\begin{array}{l}0 \\
0 \\
0\end{array}$ & $\begin{array}{l}0 \\
0 \\
0\end{array}$ & E & $\begin{array}{l}5 \\
1 \\
4\end{array}$ & $\begin{array}{r}157 \\
67 \\
235\end{array}$ & $\frac{(51-366)}{(64-602)}$ & $\begin{array}{l}66 \\
42 \\
24\end{array}$ & $\begin{array}{r}108 \\
95 \\
142\end{array}$ & $\begin{array}{l}(83-137) \\
(68-128) \\
(91-211)\end{array}$ \\
\hline $\begin{array}{l}\text { Colon } \\
\text { (153) }\end{array}$ & $\begin{array}{l}\text { combined } \\
\text { retired } \\
\text { active }\end{array}$ & $\begin{array}{l}0 \\
0 \\
0\end{array}$ & $\begin{array}{l}0 \\
0 \\
0\end{array}$ & $=$ & $\begin{array}{l}0 \\
0 \\
0\end{array}$ & $\begin{array}{l}0 \\
0 \\
0\end{array}$ & $\bar{z}$ & $\begin{array}{l}47 \\
34 \\
13\end{array}$ & $\begin{array}{l}89 \\
91 \\
85\end{array}$ & $\begin{array}{l}(66-119) \\
(63-127) \\
(45-146)\end{array}$ \\
\hline $\begin{array}{l}\text { Rectum } \\
\text { (154) }\end{array}$ & $\begin{array}{l}\text { combined } \\
\text { retired } \\
\text { active }\end{array}$ & $\begin{array}{l}0 \\
0 \\
0\end{array}$ & $\begin{array}{l}0 \\
0 \\
0\end{array}$ & $=$ & $\begin{array}{l}1 \\
0 \\
1\end{array}$ & $\begin{array}{r}72 \\
0 \\
130\end{array}$ & $\bar{z}$ & $\begin{array}{l}35 \\
24 \\
11\end{array}$ & $\begin{array}{l}127 \\
123 \\
137\end{array}$ & $\begin{array}{l}(88-177) \\
(79-183) \\
(68-245)\end{array}$ \\
\hline $\begin{array}{l}\text { Liver or gallbladder } \\
(155-156)\end{array}$ & $\begin{array}{l}\text { combined } \\
\text { retired } \\
\text { active }\end{array}$ & $\begin{array}{l}0 \\
0 \\
0\end{array}$ & $\begin{array}{l}0 \\
0 \\
0\end{array}$ & E & $\begin{array}{l}2 \\
0 \\
2\end{array}$ & $\begin{array}{r}168 \\
0 \\
298\end{array}$ & $\frac{(20-606)}{(36-1075)}$ & $\begin{array}{r}12 \\
8 \\
4\end{array}$ & $\begin{array}{l}50 \\
50 \\
52\end{array}$ & $\begin{array}{l}(26-88) \\
(21-98) \\
(14-132)\end{array}$ \\
\hline $\begin{array}{l}\text { Pancreas } \\
(157)\end{array}$ & $\begin{array}{l}\text { combined } \\
\text { retired } \\
\text { active }\end{array}$ & $\begin{array}{l}0 \\
0 \\
0\end{array}$ & $\begin{array}{l}0 \\
0 \\
0\end{array}$ & z & $\begin{array}{l}3 \\
1 \\
2\end{array}$ & $\begin{array}{l}178 \\
154 \\
193\end{array}$ & $\frac{(37-520)}{(23-697)}$ & $\begin{array}{r}19 \\
14 \\
5\end{array}$ & $\begin{array}{l}62 \\
72 \\
46\end{array}$ & $\begin{array}{l}(38-97) \\
(39-120) \\
(15-106)\end{array}$ \\
\hline $\begin{array}{l}\text { Larynx } \\
(161)\end{array}$ & $\begin{array}{l}\text { combined } \\
\text { retired } \\
\text { active }\end{array}$ & $\begin{array}{l}1 \\
0 \\
1\end{array}$ & $\begin{array}{r}1243 \\
0 \\
1291\end{array}$ & E & $\begin{array}{l}0 \\
0 \\
0\end{array}$ & $\begin{array}{l}0 \\
0 \\
0\end{array}$ & $\overline{-}$ & $\begin{array}{r}12 \\
8 \\
4\end{array}$ & $\begin{array}{r}130 \\
164 \\
91\end{array}$ & $\begin{array}{l}(67-226) \\
(71-322) \\
(25-234)\end{array}$ \\
\hline $\begin{array}{l}\text { Trachea or bronchus } \\
\text { or lung } \\
\text { (162) }\end{array}$ & $\begin{array}{l}\text { combined } \\
\text { retired } \\
\text { active }\end{array}$ & $\begin{array}{l}2 \\
0 \\
2\end{array}$ & $\begin{array}{r}213 \\
0 \\
229\end{array}$ & $\frac{(26-771)}{(28-828)}$ & $\begin{array}{r}17 \\
9 \\
8\end{array}$ & $\begin{array}{l}165 \\
218 \\
130\end{array}$ & $\begin{array}{l}(96-265) \\
(100-413) \\
(56-256)\end{array}$ & $\begin{array}{r}238 \\
154 \\
84\end{array}$ & $\begin{array}{l}129 \\
132 \\
122\end{array}$ & $\begin{array}{l}(113-146) \\
(112-155) \\
(97-151)\end{array}$ \\
\hline $\begin{array}{l}\text { Pleura } \\
(163)\end{array}$ & $\begin{array}{l}\text { combined } \\
\text { retired } \\
\text { active }\end{array}$ & $\begin{array}{l}0 \\
0 \\
0\end{array}$ & $\begin{array}{l}0 \\
0 \\
0\end{array}$ & - & $\begin{array}{l}0 \\
0 \\
0\end{array}$ & $\begin{array}{l}0 \\
0 \\
0\end{array}$ & E & $\begin{array}{r}17 \\
13 \\
4\end{array}$ & $\begin{array}{l}431 \\
555 \\
249\end{array}$ & $\begin{array}{l}(251-689) \\
(295-950) \\
(68-637)\end{array}$ \\
\hline $\begin{array}{l}\text { Prostate } \\
\text { (185) }\end{array}$ & $\begin{array}{l}\text { combined } \\
\text { retired } \\
\text { active }\end{array}$ & $\begin{array}{l}0 \\
0 \\
0\end{array}$ & $\begin{array}{l}0 \\
0 \\
0\end{array}$ & $\overline{-}$ & $\begin{array}{l}2 \\
2 \\
0\end{array}$ & $\begin{array}{r}109 \\
149 \\
0\end{array}$ & $\begin{array}{l}(13-392) \\
(18-537) \\
-\end{array}$ & $\begin{array}{r}68 \\
60 \\
8\end{array}$ & $\begin{array}{r}107 \\
109 \\
99\end{array}$ & $\begin{array}{l}(83-136) \\
(83-140) \\
(42-194)\end{array}$ \\
\hline $\begin{array}{l}\text { Bladder } \\
\text { (188) }\end{array}$ & $\begin{array}{l}\text { combined } \\
\text { retired } \\
\text { active }\end{array}$ & $\begin{array}{l}0 \\
0 \\
0\end{array}$ & $\begin{array}{l}0 \\
0 \\
0\end{array}$ & $\overline{-}$ & $\begin{array}{l}3 \\
2 \\
1\end{array}$ & $\begin{array}{l}281 \\
325 \\
220\end{array}$ & $\begin{array}{l}(58-820) \\
(39-1175) \\
-\end{array}$ & $\begin{array}{r}32 \\
26 \\
6\end{array}$ & $\begin{array}{l}118 \\
122 \\
106\end{array}$ & $\begin{array}{l}(81-167) \\
(80-178) \\
(39-231)\end{array}$ \\
\hline $\begin{array}{l}\text { Brain or central } \\
\text { nervous system } \\
(191-192)\end{array}$ & $\begin{array}{l}\text { combined } \\
\text { retired } \\
\text { active }\end{array}$ & $\begin{array}{l}0 \\
0 \\
0\end{array}$ & $\begin{array}{l}0 \\
0 \\
0\end{array}$ & E & $\begin{array}{l}1 \\
0 \\
1\end{array}$ & $\begin{array}{r}113 \\
0 \\
139\end{array}$ & E & $\begin{array}{l}8 \\
4 \\
4\end{array}$ & $\begin{array}{l}82 \\
95 \\
72\end{array}$ & $\begin{array}{l}(35-162) \\
(26-243) \\
(19-185)\end{array}$ \\
\hline $\begin{array}{l}\text { Lymphatic system } \\
(200-203)\end{array}$ & $\begin{array}{l}\text { combined } \\
\text { retired } \\
\text { active }\end{array}$ & $\begin{array}{l}0 \\
0 \\
0\end{array}$ & $\begin{array}{l}0 \\
0 \\
0\end{array}$ & E & $\begin{array}{l}3 \\
1 \\
2\end{array}$ & $\begin{array}{l}251 \\
269 \\
243\end{array}$ & $\frac{(52-733)}{(29-877)}$ & $\begin{array}{r}14 \\
6 \\
8\end{array}$ & $\begin{array}{r}70 \\
47 \\
108\end{array}$ & $\begin{array}{l}(38-117) \\
(17-103) \\
(46-212)\end{array}$ \\
\hline $\begin{array}{l}\text { Leukaemia } \\
(204-208)\end{array}$ & $\begin{array}{l}\text { combined } \\
\text { retired } \\
\text { active }\end{array}$ & $\begin{array}{l}0 \\
0 \\
0\end{array}$ & $\begin{array}{l}0 \\
0 \\
0\end{array}$ & - & $\begin{array}{l}0 \\
0 \\
0\end{array}$ & $\begin{array}{l}0 \\
0 \\
0\end{array}$ & - & $\begin{array}{r}29 \\
22 \\
7\end{array}$ & $\begin{array}{l}159 \\
171 \\
130\end{array}$ & $\begin{array}{l}(106-228) \\
(107-259) \\
(52-268)\end{array}$ \\
\hline
\end{tabular}

*On 1 January 1981.

†SMR based on the male population of western Germany as reference.

slight excess in mortality after 20 and more years (SMR 118; 95\% CI 81-167). Deaths from leukaemia occurred not earlier than 20 years after hire. After this period, there was a significant excess in mortality (SMR 159; 95\% CI 106-228) which was most pronounced among retirees (SMR 171; 95\% CI 107-259).

\section{Discussion}

This study is the first cohort study conducted on workers in the German rubber industry. Although it is one of the largest epidemiological investigations in the German industry to date about the number of cohort members and observed deaths, high data completeness and quality could be achieved. The study design, however, had to take the availability of data into account. The study cohort consisted therefore of two discrete subcohorts. The subcohort of retirees provides a vanishing opportunity to evaluate the health effects of early occupational hazards in the rubber industry in Germany. However, the members of this sub- cohort are subject to selective survival, as by definition, they had to survive until 1981. The subcohort of active workers were a cross section of all those who were alive and still employed at the beginning of follow up. These workers have also been subject to selection, because employees who died or lost their job before 1 January 1981 for whatever reason could not be included in the cohort.

The degree to which selection bias occurred in this study cannot be definitively measured, but is likely to be higher for workers with earlier years of hire. Thus, for conditions with short latency periods, and for those more rapidly fatal, the two subcohorts may partly represent those who have survived the risk period. This must be kept in mind when interpreting our findings of a lower mortality from malignant neoplasms of several sites, of which some have been associated with working conditions in the rubber industry in previous studies-for example, liver cancer, brain cancer, and lymphoma.

The lower than expected mortality from all causes among the subcohort of active workers is 
likely to be due to a healthy active worker effect, which is, as expected, not present among retirees. Correspondingly, mortality from ischaemic heart diseases and chronic obstructive pulmonary disease was lower than expected among the active workers, but increases were identified among the retirees. Interestingly, increased mortality from diabetes mellitus and hypertensive diseases had also been found in previous studies. ${ }^{15} 19$ It is unclear whether these associations are due to occupational exposures or a reflection of health behaviour and other risk factors.

Despite the potential for selective survival, excesses of deaths from cancers of several sites were identified. The most striking findings of this study were the excess deaths from pleural cancer. These entirely occurred more than 20 years after hire. Mesothelioma was recorded as the cause of death for 16 of the 17 cases identified in this study. Necropsies, which were performed on three of these men, confirmed the diagnosis in all three cases. Mortality from pleural cancer has been reported separately from lung cancer in only one previous study, which showed a notable excess. ${ }^{11}$ The potential exposure of rubber workers to asbestos has been discussed over several years. ${ }^{29} 42$ Possible sources include asbestos contaminated talc, insulation materials, and gloves. Exposure to asbestos is the likely cause of the observed excesses.

The mortality from lung cancer was significantly increased among both retired and active workers. Cohort members who had started work after 1960 were also affected. The increase was significant after more than 20 years since hire. Several other studies have reported excess mortality and morbidity from lung cancers among workers in the rubber industry and various exposures, including vulcanising fumes, talc, and nitrosamines, have been discussed as possible causal factors. ${ }^{12812-14}$ 27-29 However, only one of the above investigations was able to adjust for smoking, which did reduce but not eliminate the observed association. ${ }^{28}$ In the present study it was not possible to control for smoking. Urban residence ${ }^{43}$ is unlikely to explain our findings, because the residence of cohort members was evenly divided between urban and rural areas. Although the excess in mortality was significant, the SMRs were of a magnitude which cannot rule out confounding by smoking. ${ }^{44}$

Concern about an increased risk of laryngeal cancers was one of the reasons for this study. Our findings, however, do not provide conclusive evidence so far. Thirteen deaths from laryngeal cancer were found during the observation period. This is higher than expected, but the increase is not significant. Stratification by cohort status, year of hire, and years since hire does not yield a clearer picture. A similar finding emerged for the mortality from cancer of the pharynx. It seems that the risk of both cancers was increased in retirees who were hired before 1960, but an increase, although not significant, was also seen among active workers who started to work after 1960. This inconsistent pattern may be attributed to chance, as numbers were small. It may also reflect more complex mechanisms, such as selective survival among retirees and early retirement due to health problems. However, personal behaviour, particularly smoking and alcohol consumption, are important risk factors for cancer of both sites, ${ }^{45-47}$ but could not be recorded in the present study. Nevertheless, although confounding cannot be ruled out, excesses of cancer of the larynx, ${ }^{10-134548}$ and of the oral cavity and pharyn $x^{1142548}$ have also been found in other studies on workers in the rubber industry. Factors which have been discussed as possible causes include exposure to dust and solvents. ${ }^{10-13}$ Thus, occupational factors remain a plausible explanation for the observed excess deaths from these two cancers in the present study.

The mortality from stomach cancer in this study cohort was, although not significant, higher than expected from the national rates. Excess deaths occurred only among the subcohort of active workers and those who started work after 1960. Increased mortality from stomach cancer among workers in the rubber industry has been found in other studies $^{2} 10121519$ and has been associated with dust exposure, especially in the area of compounding, mixing, and milling. ${ }^{812} 2949$

The finding of a significant excess mortality from bladder cancer among workers hired since 1960 was unexpected. The fact that this excess was found in both subcohorts may argue against a chance finding. The occupationally induced risk of bladder cancer in the rubber industry is usually associated with exposure to aromatic amines, which resulted mainly from the use of certain antioxidants contaminated with about $0 \cdot 2 \%$ of $\beta$-naphthylamine..$^{29} 3451$ After warnings in 1949 and the early 1950s these ingredients were withdrawn and an elimination of the excess risk since then has been reported for the British rubber industry. ${ }^{12} 34$ 50-53 However, at least in Germany small quantities of $\beta$-naphthylamine remained in some of the ingredients until the mid1960 s. Thus, there is a possibility that aromatic amines or some other yet unknown occupational factor may have played a part in our findings. A chance finding or confounding by another risk factor for bladder cancer also cannot be ruled out. Due to lack of nationwide cancer registration, as is available for example in the United Kingdom, we could not study tumour incidence which would have been desirable, as the survival of patients with tumours of the bladder has improved in recent years. ${ }^{52-54}$

Excess deaths from leukaemia have been found in three other epidemiological studies in the rubber industry. ${ }^{71524}$ An association with exposure to solvents is widely accepted. ${ }^{1629556}$ Solvents, such as benzene, have been used in the study plants at least until the early 1950 s. In our study, a significant increase in the mortality from leukaemia was only found among those hired between 1950 and 1959. It may be that these men had particularly high exposures to 
solvents, but this needs to be confirmed by further analyses of the work histories, before other occupational or non-occupational hazards can be ruled out.

\section{Conclusion}

In this paper we report the total and cause specific mortality of German men in the rubber industry between 1981 and 1991 in comparison with the respective national reference rates. We present the cancer specific mortalities among workers who were retired or active on 1 January 1981, by year of hire and years since hire. Significant excesses in mortality from pleural cancer, lung cancer, and leukaemia were found in the combined cohort or one of the two subcohorts. Excess deaths, although not significant, from cancer of other sites, including the pharynx, larynx, stomach, and bladder, were identified. Thus, mortalities from cancer of several sites previously associated with the rubber industry were also increased in workers of the German rubber industry. Results of the stratified analyses are consistent with a role of occupational exposure in the aetiology of some of these cancers. We are currently conducting analyses of the work history data to find whether any of the observed excesses of cancer deaths are related to work areas and specific exposures.

We thank the participating companies, the health insurance companies (Betriebskrankenkassen-BKK), the population registries, and the community health departments (especially Dr K istries, and the community health departments (especially Dr K Plentz and Professor P Volk) all of whom contributed tremen-
dously during the data gathering phase of this study. Without dously during the data gathering phase of this study. Without
their help this study could not have been completed. We are particularly grateful to Professor $\mathrm{K}$ Norpoth and Dr CA Veys for their continuing support and scientific advice throughou the study. Finally we acknowledge the important contributions to this study from field workers, programmers, and secretaries. The study was funded by the German Federal Ministry of Research and Technology, Bonn (Förderkenn-zeichen: 01 HK470), and was initiated, funded, and supported by th Berufsgenossenschaft der Chemischen Industrie, Heidelberg.

1 Baxter PJ, Werner JB. Mortality in the British rubber industries 1967-76. London: HMSO, 1980.

2 Bovet $\mathrm{P}$, Lob $\mathrm{M}$. La mortalité par tumeur maligne chez les ouvriers d'une fabrique de caoutchouc en Suisse. Schweiz Med Wochenschr 1980;110:1277-87.

3 Parkes HG, Veys CA, Waterhouse JAH, Peters A. Cancer mortality in the British rubber industry. $\mathrm{Br} \mathcal{F}$ Ind Med 1982;39:209-20.

4 Kilpikari I, Pukkala E, Lehtonen M, Hakama M. Cancer incidence among Finish rubber workers. Int Arch Occup Environ Health 1982;51:65-71.

5 Kilpikari I. Mortality among male rubber workers in Finland. Arch Environ Health 1982;37:295-9.

6 Holmberg B, Westerholm P, Maasing R, Kestrup L, Gumaelius K, Holmlund L, Englund A. Retrospective cohort study of two plants in the Swedish rubber induscohort study of two plants in the Swedish rubber

7 Norseth T, Andersen A, Giltvedt J. Cancer incidence in the rubber industry in Norway. Scand $\mathcal{F}$ Work Environ Health 1983;9:69-71.

8 Gustavsson P, Hogstedt C, Holmberg B. Mortality and incidence of cancer among Swedish rubber workers 1952-1981. Scand $f$ Work Environ Health 1986;12: 538-44.

9 Sorahan T, Parkes HG, Veys CA, Waterhouse JAH Cancer mortality in the British rubber industry 1946-80. BrF Ind Med 1986;43:363-73.

10 Bernardinelli L, Marco RD, Tinelli C. Cancer mortality in an Italian rubber factory. $\mathrm{Br} \mathcal{F}$ Ind $\mathrm{Med}$ 1987;44:187-91.

11 Negri E, Piolatto G, Pira E, Decarli A, Kaldor J, La Vecchia C. Cancer mortality in a northern Italian cohort of rubber workers. Br f Ind Med 1989;46:624-8.

12 Sorahan T, Parkes HG, Veys CA, Waterhouse JAH, Straughan JK, Nutt A. Mortality in the British rubber Straughan JK, Nutt A. Mortality in the British

13 Szeszenia-Daborowska N, Wilczynska U, Kaczmarek T, Szymczak W. Cancer mortality among male workers in Szymczak W. Cancer mortality among male workers in
the Polish rubber industry. Pol $₹$ Occup Med Environ the Polish rubber ind
Health 1991;4:149-57.
14 Solionova LG, Smulevich VB. Mortality and cancer incidence in a cohort of rubber workers in Moscow. Scand $\mathcal{F}$ Work Environ Health 1993;19:96-101.

15 McMichael AJ, Spirtas R, Kupper LL. An epidemiologic study of mortality within a cohort of rubber workers, 1964-72. 7 Occup Med 1974;16:458-64.

16 McMichael AJ, Spirtas R, Kupper LL, Gamble JF. Solvent exposure and leukemia among rubber workers: an epidemiologic study. ₹ Occup Med 1975;17:234-9.

17 McMichael AJ, Andjelkovic DA, Tyroler, HA. Cancer mortality among rubber workers: an epidemiologic study. Ann NY Acad Sci 1976;271:125-37.

18 McMichael AJ, Spirtas R, Gamble JF, Tousey PM. Mortality among rubber workers: relationship to specific jobs. $\mathcal{F}$ Occup Med 1976;18:178-85.

19 Andjelkovich D, Taulbee J, Symons M. Mortality experience of a cohort of rubber workers, 1964-73. F Occup Med 1976;18:387-94

20 Monson RR, Nakano KK. Mortality among rubber workers. I. White male union employees in Akron, Ohio. $\mathrm{Am} \mathcal{F}$ Epidemiol 1976;103:284-96.

21 Monson RR, Nakano KK. Mortality among rubber workers. II. Other employees. Am $\mathcal{F}$ Epidemiol 1976;103: 297-303.

22 Andjelkovich D, Taulbee J, Symons $M$, Williams $T$. Mortality of rubber workers with reference to work experience. F Occup Med 1977;19:397-405

23 Andjelkovich D, Taulbee J, Blum S. Mortality of female workers in a rubber manufacturing plant. $\mathcal{F}$ Occup Med 1978;20:409-13.

24 Delzell E, Monson RR. Mortality among rubber workers. III. Cause-specific mortality, 1940-78. F Occup Med 1981;23:677-84.

25 Delzell E, Louik C, Lewis J, Monson R. Mortality and morbidity among workers in the rubber and tire industry. Am f Ind Med 1981;2:209-16.

26 Carlo GL, Jablinske MR, Lee NL, Sund KG, Corn M. Reduced mortality among workers at a rubber plant. $\mathcal{f}$ Occup Med 1993;35:611-6.

27 Wang HW, You XJ, Qu YH, Wang WF, Wang DA, Long YM, Ni JA. Investigation of cancer epidemiology and study of carcinogenic agents in the Shanghai rubber industry. Cancer Res 1984;44:3101-5.

28 Zhang ZF, Yu SZ, Li WX, Choi BCK. Smoking, occupational exposure to rubber and lung cancer. $\mathrm{Br} \mathcal{F}$ Ind Med $1989 ; 46: 12-5$.

29 IARC. IARC monographs on the evaluation of carcinogenic risk of chemicals to humans. The rubber industry. Volume 28. Lyon: IARC, 1982

30 IARC. IARC monographs on the evaluation of carcinogenic risk of chemicals to humans. Overall evaluations of carcinogenicity: an updating of IARC Monographs, volumes 1-42, suppl 7. Lyon: IARC, 1987.

31 Fishbein L. Chemicals used in the rubber industry. An overview. Scand $\mathcal{F}$ Work Health 1983;9(suppl):7-14.

32 Nutt A. Rubber work and cancer-past, present and perspectives. Scand $f$ Work Environ Health 1983;9 (suppl):49-57.

33 Spiegelhalder B, Preussmann R. Occupational nitrosamine exposure. 1. Rubber and tyre industry. Carcinogenesis 1983;4:1147-52.

34 Veys CA. The rubber industry: reflections on health risks. In: AW Gardner, ed. Current approaches to occupational health 2. Bristol: Wright PSG, 1982:1-29.

35 Kromhout HH, Heederik D. Occupational epidemiology in the rubber industry: implications of exposure variability. Am ₹ Ind Med 1995;27:171-85.

36 Keil U, Weiland SK, Birk T, Spelsberg A. Epidemiologic assessment of risks for work related diseases. Soz Praeventivmed 1992;37:50-63. (In German.)

37 Birk T, Weiland, SK, Schumann J, Person M, Mundt K, Keil U. Historical cohort study in the German rubber industry: objectives, study design and data collection. Soz Praeventivmed 1995;40:135-45. (In German.)

38 Gilbert ES. Some confounding factors in the study of mortality and occupational exposures. Am 7 Epidemiol 1982; 116:177-88.

39 Selikoff IJ. Influence of age at death on accuracy of death certificate disease diagnosis: findings in $\mathbf{4 7 5}$ consecutive certificate disease diagnosis: findings in 475 consecutive Am $\Im$ Ind Med 1992;22:505-10.

40 Ron E, Carter R, Jablon S, Mabuchi K. Agreement between death certificate and autopsy diagnoses among atomic bomb survivors. Epidemiology 1994;5:48-56.

41 Breslow NE, Day NE. Statistical methods in cancer research. Vol 2. The design and analysis of cohort studies. LARC Scientific Publications 1987;82:69-71.

42 Fischbein A. Cancer mortality in a northern Italian cohort of rubber workers [correspondence]. Br f Ind Med 1990;47: 71-2.

43 Veys CA. Towards causal inference in occupational cancer epidemiology-I. An example of the interpretive value of using local rates as the reference statistic. Ann Occup Hyg 1990;34:349-59.

44 Axelson 0 . Aspects on confounding in occupational health epidemiology. Scand $\mathcal{f}$ Work Environ Health 1978;4: 85-89.

45 Muskat JE, Wynder EL. Tobacco, alcohol, asbestos, and occupational risk factors for laryngeal cancer. Cancer 1992;69:2244-51.

46 Blot WJ, McLaughlin JK, Winn DM, Austin DF, Greenberg RS, Preston-Martin S, et al. Smoking and drinking in relation to oral and pharyngeal cancer. Cancer Res 1988;48:3282-7. 
47 Huebner WW, Schoenberg JB, Kelsey JL, Wilcox HB, McLaughlin JK, Greenberg RS, et al. Oral and pharyngeal cancer and occupation: a case-control study. Epidemiology 1992;3:300-9.

48 Norell S, Ahlbohm A, Lipping H, Österblom L. Oesophageal cancer and vulcanisation work. Lancet 1993;26:462-3.

49 Coggon D, Barker DJP, Cole RB. Stomach cancer and work in dusty trades. Br $\mathcal{Y}$ Ind Med 1990;47:298-301.

50 Veys CA. Bladder cancer in rubber workers: the story reviewed and updated. Plastics Rubber Process Applications reviewed and upd

51 Veys CA. Bladder cancer as an occupational disease in the British rubber industry: an in depth factory study to show the extent of the risk and confirmation of its subsequent disappearance. Progress in Rubber and Plastics Technology 1992;8:1-14.

52 Sorahan T. Bladder tumours among UK rubber workers [letter]. Ann Occup Hyg 1994;1:103-4.

53 Veys CA. Bladder tumours among UK rubber workers [letter]. Ann Occup Hyg 1994;1:105-6.

54 Veys CA. Towards causal inference in occupational cancer epidemiology-II. Getting the count right. Ann Occup Hyg 1993;37:181-9.

55 Arp EW, Wolf PH, Checkoway H. Lymphocytic leukemia and exposures to benzene and other solvents in the rubber and exposures to benzene and other solvents

56 Checkoway $H$, Wilcosky $T$, Wolf $P$, Tyroler $H$. An evaluation of the associations of leukemia and rubber industry solvent exposures. Am $\mathcal{F}$ Ind Med 1984;5:239-49.

\section{Vancouver style}

All manuscripts submitted to Occup Environ Med should conform to the uniform requirements for manuscripts submitted to biomedical journals (known as the Vancouver style.)

Occup Environ Med, together with many other international biomedical journals, has agreed to accept articles prepared in accordance with the Vancouver style. The style (described in full in the BMf, 24 February 1979, p 532) is intended to standardise requirements for authors.

References should be numbered consecutively in the order in which they are first mentioned in the text by Arabic numerals above the line on each occasion the reference is cited (Manson ${ }^{1}$ confirmed other reports $^{2-5} \ldots$.). In future references to papers submitted to Occup Environ Med should include: the names of all authors if there are seven or less or, if there are more, the first six followed by et al; the title of journal articles or book chapters; the titles of journals abbreviated according to the style of Index Medicus; and the first and final page numbers of the article or chapter. Titles not in Index Medicus should be given in full.

Examples of common forms of references are:

1 International Steering Committee of Medical Editors, Uniform requirements for manuscripts submitted to biomedical journals. Br Med f 1979;1:532-5.

2 Soter NA, Wasserman SI, Austen KF. Cold urticaria: release into the circulation of histamine and eosinophil chemotactic factor of anaphylaxis during cold challenge. N Engl $f$ Med 1976;294:687-90.

3 Weinstein $\mathrm{L}$ Swartz MN. Pathogenic properties of invading mwart $\mathrm{MN}$. Pathogenic properties of Sademan Wr, Sodeman WA, eds. Pathologic physiology, mechanisms 